\title{
Automatic Whole Heart Segmentation in Static Magnetic Resonance Image Volumes
}

\author{
Jochen Peters, Olivier Ecabert, Carsten Meyer, Hauke Schramm^, \\ Reinhard Kneser, Alexandra Groth, and Jürgen Weese \\ Philips Research Laboratories, X-Ray Imaging Systems, \\ Weisshausstrasse 2, D-52066 Aachen, Germany \\ \{jochen.peters, carsten.meyer\}@philips.com \\ ${ }^{\star}$ FH Kiel, Sokratesplatz 1, D-24149 Kiel, Germany
}

\begin{abstract}
We present a fully automatic segmentation algorithm for the whole heart (four chambers, left ventricular myocardium and trunks of the aorta, the pulmonary artery and the pulmonary veins) in cardiac MR image volumes with nearly isotropic voxel resolution, based on shape-constrained deformable models. After automatic model initialization and reorientation to the cardiac axes, we apply a multi-stage adaptation scheme with progressively increasing degrees of freedom. Particular attention is paid to the calibration of the MR image intensities. Detailed evaluation results for the various anatomical heart regions are presented on a database of 42 patients. On calibrated images, we obtain an average segmentation error of $0.76 \mathrm{~mm}$.
\end{abstract}

\section{Introduction}

Cardiovascular disease is the leading direct or contributing cause of death in the world. Three-dimensional (3-D) magnetic resonance (MR) imaging is now well established in the noninvasive diagnosis of cardiovascular disease. A prerequisite to image interpretation, e.g. 3-D visualization, is image segmentation. Due to the increasing amount of image data associated with finer resolution, segmentation needs to be highly automated to be clinically valuable. Automatic segmentation of cardiac MR images is however challenging due to image noise, low tissue contrast between the myocardium and surrounding tissues, patient variability, the lack of gray level calibration and spatial magnetic field inhomogeneities.

A number of techniques have been applied to cardiac MR image segmentation. Among these are active shape models [1], active appearance models 34,5], deformable models 6 6789], active contours [10], level sets [11, and atlas-based methods 12. While many studies focus on segmenting only the left and right ventricles and the myocardium, segmentations of the four chambers plus the left ventricular myocardium have recently been reported in [131114. Often, dynamic MR images are acquired in a stack of slices in short axis and/or long axis views, and consequently the algorithms are developed for this kind of data. Here, we focus on static cardiac (3-D) image volumes with nearly isotropic voxel resolution, acquired with steady-state free-precession MRI. For this kind of data,

N. Ayache, S. Ourselin, A. Maeder (Eds.): MICCAI 2007, Part II, LNCS 4792, pp. 402-410, 2007.

(C) Springer-Verlag Berlin Heidelberg 2007 
the heart position and axes are not known a priori and surrounding structures are visible since the field of view is not confined to the heart itself.

In this work, we outline and evaluate a fully automatic algorithm for whole heart segmentation in cardiac MR image volumes, based on shape-constrained deformable models [7. In previous work, we have successfully applied this framework to cardiac CT images [15]. Here, particular attention is paid to a novel model initialization step as well as to the effect of MR image calibration to compensate for image intensity variations [16174]. Our main contributions are:

- fully automatic heart localization based on a 3-D generalized Hough transformation, streamlined for fast processing,

- the evaluation of the effect of MR image calibration for the given task,

- the demonstration of fully automatic whole heart segmentation (four chambers, left ventricular myocardium and trunks of aorta, pulmonary artery and pulmonary veins) in nearly isotropic, static cardiac MR image volumes.

\section{Shape-Constrained Deformable Models}

In this work, the cardiac anatomy is extracted from the MR image volumes using a deforming mesh comprising both ventricles, both atria, the epicardial surface around the left ventricle and the trunks of the great vessels (aorta, pulmonary artery and veins, and vena cava). This mesh is made of $V=7286$ vertices combined in $T=14771$ triangles with complex junctions connecting 3 or more surfaces [18. An illustration of this mesh is given in Figure 1]

The mesh adaptation is performed by iterating two steps. First, boundary candidates are searched along the normal vectors $\boldsymbol{n}_{i}$ of the mesh triangles. For each triangle $i$, these target points are selected according to

$$
\boldsymbol{x}_{i}^{\text {target }}=\boldsymbol{c}_{i}+\underset{j=-l, \ldots,+l}{\arg \max }\left[F_{i}\left(\boldsymbol{c}_{i}+j \delta \boldsymbol{n}_{i}\right)-D j^{2} \delta^{2}\right] \cdot \delta \cdot \boldsymbol{n}_{i},
$$

where $\boldsymbol{c}_{i}$ are the triangle centers, $\delta$ is the sampling distance along the normal vector, $l \cdot \delta$ is the search range and $D$ is a heuristic penalty term, which biases the search to nearby points. The choice for optimal, spatially varying boundary detection functions $F_{i}($.$) is essential for a robust and accurate segmentation. A$ more detailed discussion about these functions is provided in Section 4.

In the second step, the mesh is deformed by minimizing the sum of the quadratic distances between the triangle centers $\boldsymbol{c}_{i}$ and the detected boundary points $\boldsymbol{x}_{i}^{\text {target }}[7]$

$$
E_{\text {external }}=\sum_{i=1}^{T} \tilde{w}_{i}\left(\frac{\nabla I\left(\boldsymbol{x}_{i}^{\text {target }}\right)}{\left\|\nabla I\left(\boldsymbol{x}_{i}^{\text {target }}\right)\right\|} \cdot\left(\boldsymbol{x}_{i}^{\text {target }}-\boldsymbol{c}_{i}\right)\right)^{2} .
$$

The term $\left(\boldsymbol{x}_{i}^{\text {target }}-\boldsymbol{c}_{i}\right)$ is projected onto the direction of the image intensity gradient $\nabla I /\|\nabla I\|$ at the target point. This makes the energy invariant 

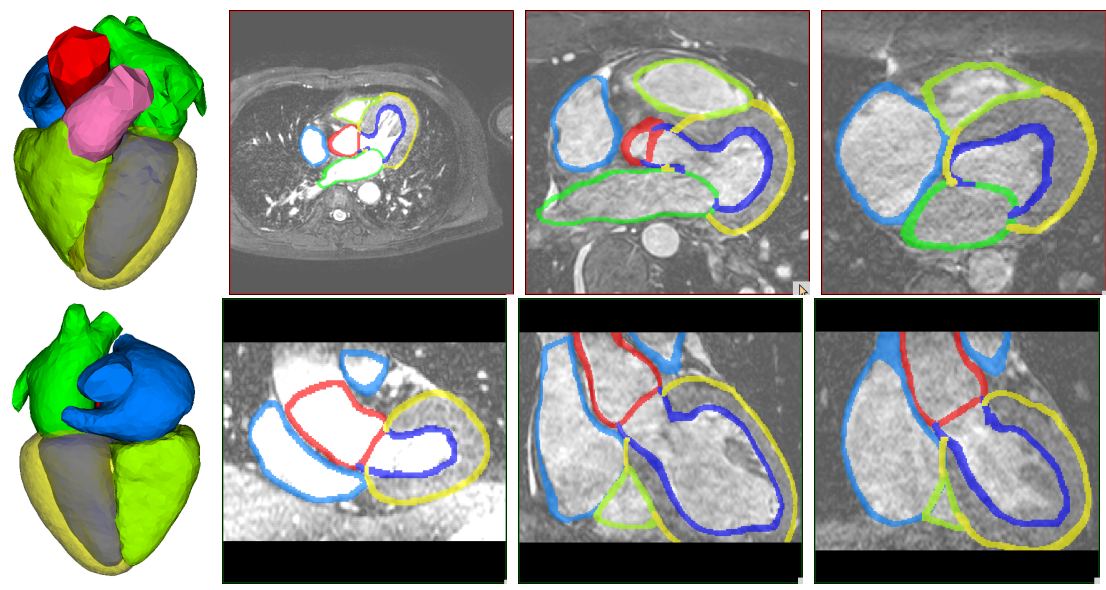

Fig. 1. Heart model from two different views, and typical results of the fully automatic segmentation (with calibration) for three different patients (first row: axial views, second row: coronal views; different zoom factors). Note the differences in contrast and relative chamber sizes and orientations.

to movements of the triangle within the object tangent plane, preventing the triangle from becoming stuck at the target position. The weights $\tilde{w}_{i}$ reflect the reliability of the detected boundary. Practically, they are set to

$$
\tilde{w}_{i}=\max \left\{0, F_{i}\left(\boldsymbol{x}_{i}^{\text {target }}\right)-D \cdot\left(\boldsymbol{x}_{i}^{\text {target }}-\boldsymbol{c}_{i}\right)^{2}\right\}
$$

Minimizing Eq. (2) with respect to $\boldsymbol{c}_{i}$ may lead to irregular shapes due to some false target points and missing boundaries $\left(\tilde{w}_{i}=0\right)$. Prior shape knowledge can be used to stabilize this problem, as described in the following.

Shape-Constrained Deformable Adaptation. To constrain the deformation we require the vertices $\boldsymbol{v}_{i}$ to stay close to those of a reference shape (e.g., mean shape $\boldsymbol{m}$ ) that is allowed to be modified by a geometric transformation $\mathcal{T}$ [.] (e.g., rigid or affine). This can be formalized by introducing an internal energy

$$
E_{\text {internal }}=\sum_{i=1}^{V} \sum_{j \in N(i)}\left(\left(\boldsymbol{v}_{i}-\boldsymbol{v}_{j}\right)-\left(\mathcal{T}\left[\boldsymbol{m}_{i}\right]-\mathcal{T}\left[\boldsymbol{m}_{j}\right]\right)\right)^{2},
$$

where $N(i)$ is the set of indices of the first-order neighbor vertices of vertex $\boldsymbol{v}_{i}$. The objective function to minimize is now given by $E=E_{\text {external }}+\alpha E_{\text {internal }}$ 7], where $\alpha$ balances the contribution of the internal and external energy. The free variables are the vertex positions $\boldsymbol{v}_{i}$ and the transformation parameters of $\mathcal{T}$. The boundary detection step followed by the optimization of $E$ is iterated until the mesh reaches a steady state. 


\section{Automatic Whole Heart Segmentation}

To achieve robust segmentation, we use a multi-stage adaptation scheme:

1. Heart Localization. The first step of the segmentation chain consists of detecting the heart using the generalized Hough transformation (GHT) [19]. GHT is a method to detect the occurrence of any characteristic shape undergoing a geometric transformation in a 2-D image. It can be straightforwardly extended to 3-D, but the computational complexity and memory demand make this method inappropriate. In this paper, we introduce a practicable solution that makes use of image properties and characteristics of the cardiac anatomy. In particular, we constrain the geometric transformation to translation and scaling.

We can observe that the heart is much larger than the voxel resolution. Before locating the heart in a new image, we can first down-sample the input image to voxel resolution $3.0 \times 3.0 \times 3.0 \mathrm{~mm}^{3}$. After this step, the global shape of the heart is still well preserved while small surrounding structures are suppressed.

Then, we filter out many disturbing edges by applying a threshold on the image to coarsely separate the blood pool from the remaining structures. GHT is then performed on the edge image resulting after this thresholding operation. That is, we actually do not directly search for the heart in the image, but indirectly by localizing the blood pool which has better contrast in our MR images.

GHT is usually trained for one single reference shape of an object class. We can learn the shape variability of several individuals by combining the R-tables of several reference shapes [17. After encoding the shapes from various patients, we can prune the R-table and keep only those entries that occur more than $\tau$ times (in our experiments $\tau=2$ ). That way, the entries are more discriminative and the detection time is reduced.

2. Parametric Adaptation. After heart localization by the GHT, the model might still be "far" from the heart boundaries which increases the risk of false target points. This motivates to first adapt the heart model in a parametric instead of a fully deformable way. In particular, the global pose has yet to be refined (i.e., up to now the model has not been rotated). The parametric adaptation works as follows: We apply a geometric transformation $\mathcal{T}$ [.] to the whole model and use the same transformation in Eq. (3). $E_{\text {internal }}$ thus vanishes and we optimize $E_{\text {external }}$ alone. Assuming that $\mathcal{T}$ [.] can be described by some parameters $\boldsymbol{q}=\left(q_{1} \ldots q_{M}\right)^{\top}$ and that the transformation is applied to the whole mesh, the vertex positions can be expressed as $\boldsymbol{v}(\boldsymbol{q})$. The goal is now to find the parameters $\boldsymbol{q}$ that minimize $E_{\text {external }}(\boldsymbol{v}(\boldsymbol{q}))$. The boundary detection step followed by the optimization of the energy function is iterated until convergence.

The geometric transformation $\mathcal{T}[$.$] is successively refined with increasing de-$ grees of freedom (number of parameters) $\boldsymbol{q}$, starting with a global similarity transformation $\left(\mathcal{T}[]=.\mathcal{T}_{\text {similarity }}[].\right)$ for global pose correction. Then, to correct for anisotropic scale variations, we use a global affine transformation $\left(\mathcal{T}[]=.\mathcal{T}_{\text {affine }}[].\right)$. Finally, we assign an individual affine transformation to each of the $K$ anatomical regions of the model to capture changes in size and rotation 
of the chambers between patients. To ensure that the mesh remains smooth at the transitions between two (or more) anatomical regions, we linearly interpolate the affine transformations as

$$
\mathcal{T}_{\text {piecewise affine }}\left[\boldsymbol{v}_{i}\right]=\sum_{k=1}^{K} w_{i, k} \cdot \mathcal{T}_{\text {affine }, k}\left[\boldsymbol{v}_{i}\right],
$$

where $w_{i, k}$ is the contribution of transformation $\mathcal{T}_{\text {affine, } k}[$.$] for vertex i$, satisfying the normalization condition $\sum_{k=1}^{K} w_{i, k}=1 \forall i$.

3. Deformable Adaptation. The model is now usually initialized well enough to proceed with a deformable adaptation as described in Section 2, i.e., the model is no longer constrained to undergo the transformation $\mathcal{T}$ [.]. Here, we keep using the piecewise affine transformation Eq. (4) in the internal energy Eq. (3).

In summary, the degrees of freedom of the mesh deformation are progressively increased during segmentation, thereby increasing the robustness of the segmentation.

\section{Optimized Boundary Detection}

Vertex correspondence is preserved during mesh adaptation. This property enables us to encode information into the model which will be carried with the triangles during the adaptation. Especially, in a training phase a locally optimal boundary detection function is assigned to each mesh triangle using the Simulated Search approach [20].

This method needs as input adapted reference meshes and candidates of boundary detection functions. The selection process works as follows for each triangle independently:

1. The pose of the reference triangles is slightly disturbed, i.e., the triangle is shifted (along the normal vector and/or laterally) and/or tilted.

2. The boundary detection of Eq. (1) is performed.

3. The residual error between the detected point and the reference position is recorded for all tested displacements and all function candidates.

4. The candidate with the smallest simulated residual error is finally selected.

As function candidates, we use the magnitude of the image gradient projected onto the triangle normal vector jointly with some boundary discrimination represented by rejection criteria $Q_{k}$ and acceptance intervals $\left[\min _{k}, \max _{k}\right]$ :

$$
F_{i}(\boldsymbol{x})=\left\{\begin{array}{cl}
\left\|\boldsymbol{n}_{i} \cdot \nabla I(\boldsymbol{x})\right\| & \text { if } Q_{k} \in\left[\min _{k}, \max _{k}\right] \text { for all } Q_{k} \\
0 & \text { if } Q_{k} \notin\left[\min _{k}, \max _{k}\right] \text { for some } Q_{k}
\end{array} .\right.
$$

Rejection Criteria and Acceptance Intervals. For edge characterization, we use the following rejection criteria: the gray values on either side of the boundary and the (signed) difference of the gray values across the boundary. All these criteria can be used alone or in combination to build numerous candidates (5). 
Reasonable acceptance intervals are trained by clustering the rejection criteria introduced above into $M$ classes using the $Q_{k}$ values obtained from the reference meshes. The acceptance intervals are then derived from these clusters by rejecting the low and high $N \%$ percentiles per $Q_{k}$. Note that both $M$ and $N$ are associated with tradeoffs. Using more clusters (large $M$ ) may be more specific for the local image properties but may lead to less robust estimates. Narrow intervals (large $N$ ) might reject too many correct edges, whereas wide intervals might be too unspecific. Here, as in CT [20], we use $M=5,10$ and $N=5,10 \%$.

Finally, the candidates that result from combining all possible rejection criteria and all trained acceptance intervals are used by the Simulated Search to select the optimal boundary detection function for each triangle.

Image Intensity Calibration. To compensate for image intensity variations across images, we compute the image histogram and derive the low and high $L \%$ percentiles, similar to [16]. The intensity values within this interval are then linearly re-scaled to a reference interval computed, e.g., from a reference image. This calibration strongly reduces the intensity variations between images and yields more consistent and narrow acceptance intervals. For consistency, the image calibration has to be performed both during training and segmentation.

Preliminary experiments performed with $L=0,2,5$ and $10 \%$ show that $L=$ $2 \%$ was the value yielding the best results (i.e., smallest segmentation error) and will be used in the next section.

\section{Results}

Experimental Setup. The segmentation algorithm proposed in this paper was evaluated on 42 steady-state free-precession MR images, designated to inspect the coronary arteries specifically for ischemic disease. The images, acquired on Philips Intera and Achieva 1.5T scanners at end-diastolic phase over various cardiac cycles and breathing compensated $(\mathrm{TE}=2.14 \pm 0.11[2.01-2.38] \mathrm{ms}$, $\mathrm{TR}=4.27 \pm 0.22[4.04-4.75] \mathrm{ms}$, flip angle $=86.07 \pm 5.33[70.00-90.00]$ degrees, pixel spacing $=0.5-0.7 \mathrm{~mm}$, slice distance $=0.7-0.9 \mathrm{~mm}$, matrix size $=512 \times$ $512,100-170$ slices), were obtained from clinics in various continents.

Dividing the 42 images into 4 clusters with $N=10$ or $N=11$ images each, we used a leave- $N$-out validation approach to evaluate our segmentation algorithm. The training images were used to compute the mean mesh $\boldsymbol{m}$ (see Section 2), to generate the R-table for the GHT (see Section 3) and to train and assign the optimal boundary detection function to each triangle (see Section 4). The parameters of the algorithm $(D, \alpha, \delta$ and $l)$ have been adjusted for optimal performance on CT images; previous experiments have shown that the segmentation performance on CT images is robust with regard to these parameters.

Error Measurement. We measure the segmentation error as the symmetrized mean Euclidean "surface-to-patch" distance $\epsilon_{\text {mean }}$, i.e. the mean distance between the triangle centers of the adapted mesh to an anatomically corresponding patch of maximum geodesic radius $r=10 \mathrm{~mm}$ of the reference mesh and 
Table 1. Mean segmentation error after deformable adaptation for the different anatomical regions of the heart as well as the percentage of triangles with various error ranges, (a) without calibration, (b) with calibration.

\begin{tabular}{|c|c|c|c|c|c|c|c|c|}
\hline \multirow{3}{*}{ Anatomical region } & \multirow{2}{*}{\multicolumn{2}{|c|}{$\epsilon_{\text {mean }}(\mathrm{mm})$}} & \multicolumn{6}{|c|}{ Percentage of triangles with error range (\%) } \\
\hline & & & \multicolumn{2}{|c|}{$<1.0 \mathrm{~mm}$} & \multicolumn{2}{|c|}{$1.0-2.0 \mathrm{~mm}$} & \multicolumn{2}{|c|}{$>2.0 \mathrm{~mm}$} \\
\hline & (a) & (b) & (a) & (b) & (a) & (b) & (a) & (b) \\
\hline Whole mesh & 1.33 & $\overline{0.76}$ & $\overline{42.7}$ & $\overline{76.6}$ & $\overline{37.4}$ & 22.7 & 19.9 & $\overline{0.7}$ \\
\hline Aorta & 1.16 & $\overline{0.60}$ & 36.1 & 98.5 & 63.6 & 1.5 & 0.3 & $\overline{0.0}$ \\
\hline Left atrium & $\overline{1.44}$ & $\overline{0.72}$ & 22.8 & 80.0 & 60.7 & 18.1 & 16.5 & 1.9 \\
\hline Left ventricle & $\overline{1.59}$ & $\overline{0.69}$ & $\overline{28.6}$ & $\overline{83.2}$ & 40.5 & 16.8 & $\overline{30.9}$ & $\overline{0.0}$ \\
\hline Myocardium (LV) & 1.78 & 0.83 & 26.1 & 68.3 & 32.9 & 30.5 & 41.0 & 1.2 \\
\hline Right atrium & 0.69 & 0.63 & 83.9 & 87.7 & 15.8 & 12.3 & 0.3 & $\overline{0.0}$ \\
\hline Right ventricle & 0.95 & 0.74 & 54.9 & 81.1 & 44.0 & 18.9 & 1.1 & 0.0 \\
\hline Pulmonary artery & $\overline{0.99}$ & 0.73 & 62.9 & 78.4 & 32.8 & 21.2 & 4.3 & 0.4 \\
\hline
\end{tabular}

vice versa, averaged over all 42 data sets. The triangle positions near the artificial caps of the truncated vessels are excluded from the error measurement (1032 out of 14771 triangles), since they do not relate to anatomical structures. The reference meshes were generated semi-automatically by using preliminary boundary detection functions and a preliminary mean mesh obtained from a different database of cardiac CT images (thus unrelated to the models used in the test experiments), followed by manual correction.

Segmentation. Reasonable segmentation results have been obtained in all cases of our database. In Table 1 we evaluate the effect of calibrating training and test images according to Section 4, for the compelete mesh and each anatomical region. In particular, we show the error distribution across the triangles with the corresponding anatomical label. We find that image calibration reduces the mean segmentation error for all anatomical parts. The reduction is statistically significant (paired $t$-test) for the whole mesh, the left ventricle, and the LV myocardium $(P<0.01)$ as well as for the right chambers $(P<0.05) 11$ Furthermore, the fraction of triangles with medium to large errors $(>1 \mathrm{~mm})$ is considerably reduced, i.e., improvements are distributed over the entire model surface. For the left chambers and the myocardium, large errors $(>2 \mathrm{~mm})$ are drastically reduced. Some segmentation examples are presented in Figure 1. The whole segmentation chain needs less than a minute on a workstation with Dual-Xeon Hyper-Threading Intel processors $(2 \times 1.7 \mathrm{GHz})$ and 1 GByte RAM.

\section{Conclusion}

We have demonstrated fully automatic whole heart segmentation (four chambers, myocardium and the trunks of the aorta, pulmonary artery and pulmonary veins)

${ }^{1}$ For the remaining structures $P<0.2$. 
in static MR image volumes with nearly isotropic voxels, using shape-constrained deformable models. The 3-D generalized Hough transformation successfully localized the heart in all 42 test images. Image calibration to compensate for intensity variations in the MR images significantly improved the segmentation performance over the entire model surface. We measured a mean segmentation error of $0.76 \mathrm{~mm}$ (ranging from $0.60 \mathrm{~mm}$ for the aorta to $0.83 \mathrm{~mm}$ for the $\mathrm{LV}$ myocardium). Future work includes improved MR calibration schemes and an application of our algorithm to other imaging protocols.

Acknowledgments. For the cardiac images and fruitful discussions we are grateful to M. Breeuwer, P. Ermes, G. Hautvast and F. Gerritsen from Philips Medical Systems Healthcare Informatics, Clinical Informatics - Advanced Development (Best, The Netherlands). We also thank J. von Berg and C. Lorenz (Philips Research Hamburg) for providing the cardiac mesh.

\section{References}

1. Cootes, T.F., Taylor, C.J.: Active shape models - 'smart snakes'. In: Proc. British Machine Vis. Conf., pp. 266-275. Springer, Heidelberg (1992)

2. van Assen, H.C., Danilouchkine, M.G., Frangi, A.F., et al.: SPASM: A 3D-ASM for segmentation of sparse and arbitrarily oriented cardiac MRI data. Med. Image Anal. 10, 286-303 (2006)

3. Cootes, T.F., Edwards, G.J., Taylor, C.J.: Active appearance models. In: Burkhardt, H., Neumann, B. (eds.) ECCV 1998. LNCS, vol. 1407, pp. 484-498. Springer, Heidelberg (1998)

4. Mitchell, S.C., Lelieveldt, B.P.F., van der Geest, R.J., et al.: Multistage hybrid active appearance model matching: Segmentation of left and right ventricles in cardiac MR images. IEEE Trans. Med. Imag. 20(5), 415-423 (2001)

5. Stegmann, M.B., Pedersen, D.: Bi-temporal 3D active appearance models with applications to unsupervised ejection fraction estimation. In: Proc. SPIE Med. Imag., vol. 5747, pp. 336-350 (2005)

6. McInerney, T., Terzopoulos, D.: Deformable models in medical image analysis: a survey. Med. Image Anal. 1(2), 91-108 (2004)

7. Weese, J., Kaus, M.R., Lorenz, C., et al.: Shape constrained deformable models for 3D medical image segmentation. In: Insana, M.F., Leahy, R.M. (eds.) IPMI 2001. LNCS, vol. 2082, pp. 380-387. Springer, Heidelberg (2001)

8. Montillo, A., Metaxas, D., Axel, L.: Automated model-based segmentation of the left and right ventricles in tagged cardiac MRI. In: Ellis, R.E., Peters, T.M. (eds.) MICCAI 2003. LNCS, vol. 2878, pp. 507-515. Springer, Heidelberg (2003)

9. Kaus, M.R., Berg, J.v., Weese, J., et al.: Automated segmentation of the left ventricle in cardiac MRI. Med. Image Anal. 8, 245-254 (2004)

10. Jolly, M.P., Duta, N.D., Funka-Lea, G.F.: Segmentation of the left ventricle in cardiac MR images. In: Int. Conf. on Computer Vision, vol. 1, pp. 501-508 (2001)

11. Fritscher, K.D., Pilgram, R., Schubert, R.: Automatic cardiac 4D segmentation using level sets. In: Frangi, A.F., Radeva, P.I., Santos, A., Hernandez, M. (eds.) FIMH 2005. LNCS, vol. 3504, pp. 113-122. Springer, Heidelberg (2005)

12. Lorenzo-Valdés, M., Sanchez-Ortiz, G.I., Elkington, A.G., et al.: Segmentation of $4 \mathrm{D}$ cardiac MR images using a probabilistic atlas and the EM algorithm. Med. Image Anal. 8, 255-265 (2004) 
13. Lötjönen, J., Kivistö, S., Koikkalainen, J., et al.: Statistical shape model of atria, ventricles and epicardium from short- and long-axis MR images. Med. Image Anal. 8, 371-386 (2004)

14. Pfeifer, B., Hanser, F., Seger, M., et al.: Cardiac modeling using active appearance models and morphological operators. Medical Imaging 2005: Visualization, ImageGuided Procedures, and Display 5744, 279-289 (2005)

15. Ecabert, O., Peters, J., Walker, M.J., et al.: Automatic whole heart segmentation in CT images: Method and validation. In: SPIE-MI (2007)

16. Nyul, L.G., Udupa, J.K.: On standardizing the MR image intensity scale. Magnetic Resonance in Medicine 42, 1072-1081 (1999)

17. Brejl, M., Sonka, M.: Object localization and border detection criteria design in edge-based image segmentation: Automated learning from examples. IEEE Trans. Med. Imag. 19(10), 973-985 (2000)

18. von Berg, J., Lorenz, C.: Multi-surface cardiac modeling, segmentation, and tracking. In: Frangi, A.F., Radeva, P.I., Santos, A., Hernandez, M. (eds.) FIMH 2005. LNCS, vol. 3504, pp. 1-11. Springer, Heidelberg (2005)

19. Ballard, D.H.: Generalizing the Hough transform to detect arbitrary shapes. Pattern Recogn. 13(2), 111-122 (1981)

20. Peters, J., Ecabert, O., Schramm, H., Weese, J.: Discriminative boundary detection for model-based heart segmentation in CT images. In: SPIE-MI (2007) 